\title{
Traitement Medico-chirurgical du Mal de Pott de L'adulte. Notre Attitude au Gabon
}

\author{
Paul-Marie Loembe
}

Résumé: Sur 95 adultes porteurs d'une spondylodiscite tuberculeuse, 26 bénéficièrent d'un traitement médico-chirurgical entre 1982 et 1993. 21 présentaient des troubles neurologiques: déficit radiculaire - 4 cas, syndromes médullaires progressifs - 17 cas. L'indication opératoire fut neurologique 11 fois, mécanique pure 1 fois, étiologique 1 fois et mixte 13 fois. La voie d'abord fut antérieure ( 12 cas) ou postérieure (14 cas). La durée moyenne de suivi fut de 23 mois. Les résultats clinique et radiologique étaient satisfaisants. Le traitement médico-chirurgical présente des avantages incontestables au Gabon, vu l'importance des lésions. Au niveau du rachis cervical et lombaire, la décompression antérieure avec fusion est recommandée. Au niveau thoracique, nous intervenons le moins possible, les gibbosités gênantes étant rares; en cas d'abcès compressif, notre préférence va pour la laminotomie. La laminectomie n'est actuellement indiquée que dans les rares cas d'arachnoïdite ou de tuberculose de l'arc postérieur associées. Les indications de la biopsie chirurgicale sont précisées.

\begin{abstract}
Twenty-six of 95 adults treated for tuberculous spondylitis, between 1982 and 1993, underwent surgery. Twenty-one exhibited neurological deficits: radicular deficits: 4, and progressive spinal cord syndromes: 17 (incomplete: 13, complete, of acute onset: 4). Vertebral body compression fracture was the most prominent finding. Indications for surgery were neurologic: 11, mechanical: 1, etiologic: 1, and mixed: 13. Twelve patients had vertebrectomies, 3 laminotomies and 11 laminectomies. The average follow-up was 23 months. The neurological recovery was complete in 16 cases, partial in 4 cases and unchanged in one case. Bony consolidation occurred after 3-5 months. The medicosurgical treatment produced a very high cure rate, so rapidly, that it became the treatment of choice in our setting. Moreover, that allows to specify the diagnosis. Anterior decompression and fusion is recommended in the cervical and lumbar spine. In the thoracic segment, significant kyphosis is infrequent, so that surgical correction is rarely necessary. Laminotomy may occasionally be indicated for posterior decompression for abscess. Laminectomy is now preferred for uncommon cases of thoracolumbar posterior compression by tuberculous arachnoiditis or associated posterior vertebral tuberculosis. Indications for open biopsy are discussed.
\end{abstract}

Can. J. Neurol. Sci. 1994; 21: 339-345

Malgré l'introduction du Programme Elargi de Vaccination, la tuberculose pose encore des problèmes de santé publique en Afrique. La spondylodiscite tuberculeuse (S.D.T.) ou Mal de Pott est la plus fréquente et la plus grave des tuberculoses de l'appareil locomoteur. ${ }^{1-4}$ Son traitement reste controversé dans les pays à forte prévalence tuberculeuse, l'attitude vis à vis du foyer vertébral continuant à être partagée entre le traitement médical exclusif ${ }^{35-7}$ et le traitement médico-chirurgical. ${ }^{8-19}$

Pour notre part, disposant maintenant des structures matérielles et humaines adéquates, la présence d'une instabilité rachidienne par destruction pottique ou la survenue d'une compression radiculo-médullaire rebelle à une chimiothérapie initiale d'au moins trois semaines, conduit à une attitude interventionniste.
Nous décrivons notre expérience portant sur vingt-six cas de Mal de Pott, opérés entre Janvier 1982 et Décembre 1993.

\section{Materiel et Methodes}

De Janvier 1982 à Décembre 1993, 26 sur les 95 cas (27\%) de S.D.T. traités dans notre centre, bénéficièrent d'un traitement médico-chirurgical. La majorité des patients provenait des différentes villes ou villages du territoire gabonais, et était

\footnotetext{
From the Service de Neurochirurgie, Hôpital Fondation Jeanne Ebori, Université Omar Bongo, Libreville, Gabon.

RECEIVED DECEMBER 8, 1993. ACCEPTED IN FINAL FORM JUNE 16, 1994.

Reprint requests to: Paul-Marie Loembe. M.D., Service de Neurochirurgie. Fondation Jeanne Ebori, B.P. 212, Libreville, Gabon. Afrique Centrale.
} 
hospitalisée dans des unités diverses, qui ont été précisées dans un article précédent. ${ }^{2}$

\section{Clinique}

La série de 26 patients se composait de 9 femmes et 17 hommes, âgés de 26 à 68 ans (âge moyen: 48 ans). La durée de la symptomatologie, avant l'intervention, varia de un à douze mois (moyenne: 8 mois). Nos patients consultèrent pour douleurs rachidiennes ( 20 cas), sciatique ( 4 cas) ou brachialgie ( 2 cas), avec impotence fonctionnelle 21 fois. L'examen clinique précédant l'opération révélait des signes de déficit neurologique dans 21 cas: déficit radiculaire (4 cas), syndromes médullaires progressifs ( 17 cas) classés selon les critères de Pattisson ${ }^{7}$ (Tableau 1). Le tableau 2 précise ces derniers signes. On notait une cyphose cervicale chez deux patients (C4-C5, C5-C6) et une dorsolombaire (T12-L1) chez un autre, toutes de moins de 40 degrés (modérées). Tous les malades présentaient une altération de l'état général témoignant de l'imprégnation tuberculeuse: fébricule, amaigrissement et asthénie. Le Mal de Pott était concomittant d'une tuberculose pulmonaire active dans un cas.

Tableau 1. Examen neurologique en pre-operatoire immediat, selon la classification de Pattisson $\left(\mathrm{N}=22^{*}\right)$

\begin{tabular}{clc}
\hline Grade & Definition & $\mathbf{N}$ \\
\hline 0 & Normal & 5 \\
I & $\begin{array}{l}\text { Signes pyramidaux, sans déficit moteur } \\
\text { ni sensitif, marche sans aide. }\end{array}$ & 0 \\
Ila & $\begin{array}{l}\text { Déficit moteur partiel, pas de trouble } \\
\text { sensitif, marche avec aide. }\end{array}$ & 2 \\
Ilb & $\begin{array}{l}\text { Déficit moteur partiel, pas de trouble } \\
\text { sensitif, marche impossible. }\end{array}$ & I \\
IIl & $\begin{array}{l}\text { Déficit moteur complet, pas de trouble } \\
\text { sensitif, marche impossible. }\end{array}$ & 4 \\
IV & $\begin{array}{l}\text { Déficit moteur complet, perturbation ou } \\
\text { abolition de la sensation, marche impossible. }\end{array}$ & 6 \\
& $\begin{array}{l}\text { Déficit moteur complet, perte importante ou } \\
\text { totale de toutes les modalités sensitives, perte du }\end{array}$ & 4 \\
& contrôle sphinctérien et/ou spasmes musculaires.
\end{tabular}

Tableau 2. Signes Neurologiques $(\mathrm{N}=17)$

\begin{tabular}{ccr}
\hline Syndromes Medullaires & $\begin{array}{c}\text { Grade } \\
\text { de Pattisson }\end{array}$ & N \\
\hline PARTIELS: & & 13 \\
• tétraparésie & IIa & 2 \\
• tétraplégie & IV & 2 \\
• paraplégie & IIb & 1 \\
• paraplégie & III & 4 \\
COMPLET & IV & 4 \\
• tétraplégie** & & \\
• paraplégie** & & 4 \\
\hline
\end{tabular}

** Tétraplégie et paraplégie complètes d'aggravation brutale.
Deux de ces malades étaient drépanocytaires (forme hétérozygote AS). La notion de contage ne fut établie de façon nette que dans deux cas. Deux fois, il n'y eut pas le rappel de vaccination au B.C.G.

\section{EXAMENS COMPLEMENTAIRES}

\section{Biologie}

La vitesse de sédimentation était élevée chez tous les patients. Aucun renseignement n'a pu être retiré de l'étude de la numération globulaire et de la formule sanguine. Sur 26 intradermo-réactions (I.D.R.) à la tuberculine, 21 étaient positives, dont 8 fortement (diamètre supérieur ou égal à $18 \mathrm{~mm}$ ) et 5 réactions phlycténulaires.

Le B.K. ne fut isolé dans aucun des prélèvements effectués systématiquement dans les crachats et les urines. Les cultures du matériel de curetage (caséum et paroi) recueilli pendant l'intervention chirurgicale s'avérèrent négatives.

\section{Radiologie}

Des clichés standards du rachis furent réalisés chez tous les patients; 13 bénéficièrent d'une tomographie conventionnelle. On observait essentiellement des lésions ostéolytiques (Tableau 3). La myélographie fut pratiquée chez 21 patients (Tableau 4).

\section{Histologie}

Sur 19 prélèvements biopsiques effectués durant l'intervention chirurgicale, 15 présentaient une réaction inflammatoire de type granulomateux; les 4 autres étaient non concluants.

Tableau 3. R-X Standards $(N=26)$ / Tomographies $(N=13)$

- LESIONS OSTEOLYTIQUES CORPOREALES

- destruction: 11; écrasement: 11

- irrégularité plateaux vertébraux: 4

- géode en miroir: 1

- NOMBRE DE VERTEBRES (V) ATTEINTES:

$1 \mathrm{~V}=14 ; 2 \mathrm{~V}=11 ; 3 \mathrm{~V}=1$

- NIVEAU LESIONNEL:

- C3-C7 : 7; dorsal : 6,

- charnière dorso-lombaire : 4 ;

- lombaire : 9

Tableau 4. Myelographie $(\mathrm{N}=21)$

- Blocage complet : 13

- cervical : 4

- dorsal : 8

- lombaire : 1

- Blocage partiel : 5

- cervical : 1

- dorsal : 1

- lombaire : 3

- Compression latérale, lombaire : 3 


\section{TRAITEMENT}

\section{Médical}

Le traitement médical fut administré à tous les patients selon les recommandations de l'OMS:2 de 1982 à 1986, long régime (trithérapie: Rifampicine + Isoniazide + Ethambutol), d'une durée de 18 mois, prescrit à 8 patients; de 1987 à 1993, régime court (quadrithérapie: Rifampicine + Isoniazide + Ethambutol + Pyrazinamide) de 6 mois, prescrit à 18 patients. Cette chimiothérapie anti-tuberculeuse était initiée systématiquement trois à quatre semaines avant l'opération, sauf urgence chirurgicale (4 cas); et suivie par le Centre anti-tuberculeux de l'hôpital de Nkembo (Libreville, Gabon).

\section{Chirurgical}

L'indication opératoire était posée en présence de destructions osseuses importantes associées ou non à des troubles neurologiques, mais aussi dans certaines lésions débutantes, ou en cas de compression radiculaire ou médullaire rebelle à une chimiothérapie initiale d'au moins trois semaines. L'aggravation brutale d'une paraplégie/tétraplégie (4 cas) justifia également une décompression chirurgicale en urgence. L'indication opératoire reposa donc sur une base neurologique (décompression) 11 fois, mécanique pure (stabilisation) 1 fois, étiologique 1 fois et mixte 13 fois (neurologique et mécanique: 6 , neurologique et recherche étiologique: 4 , mécanique et recherche étiologique: 3).

\begin{tabular}{|c|c|c|c|c|}
\hline & Niveau & Description & & $\mathbf{N}$ \\
\hline \multirow{3}{*}{$\begin{array}{l}\text { Technique } \\
(N=26)\end{array}$} & $\mathrm{C} 3-\mathrm{C} 7$ & \multicolumn{2}{|l|}{$\begin{array}{l}\text { - Corp. + G+P } \\
\text { - Hogdson (H) } \\
\text { - Erad. + biopsie }\end{array}$} & $\begin{array}{l}3 \\
2 \\
2\end{array}$ \\
\hline & TI-LI & \multicolumn{2}{|l|}{$\begin{array}{l}\text { - Laminectomie } \\
\text { - Laminotomie }\end{array}$} & $\begin{array}{l}8 \\
2\end{array}$ \\
\hline & L2-L5 & \multicolumn{2}{|c|}{$\begin{array}{l}\text { - Laminectomie } \\
\text { - Laminotomie } \\
\text { - Lombotomie }(\mathrm{H}+\mathrm{A})\end{array}$} & $\begin{array}{l}3 \\
1 \\
5\end{array}$ \\
\hline \multirow{7}{*}{ Resultats } & $\begin{array}{c}\begin{array}{c}\text { Fonctionnel } \\
\text { (douleurs) } \\
N=26\end{array} \\
\end{array}$ & \multicolumn{2}{|l|}{$\begin{array}{l}\text { - disparition } \\
\text { - diminution }\end{array}$} & $\begin{array}{r}25 \\
1\end{array}$ \\
\hline & & \multicolumn{2}{|c|}{ A - Syndromes radiculaires: } & 4 \\
\hline & \multirow{4}{*}{$\begin{array}{c}\text { Neurologique } \\
\text { (récupération) } \\
n=21\end{array}$} & \multicolumn{2}{|c|}{ B - Syndromes médullaires: } & 17 \\
\hline & & $\begin{array}{l}\text { - complète/quasi: } \\
\text { - grade } 0: 6 \\
\text { - grade I: } 6\end{array}$ & 12 & \\
\hline & & $\begin{array}{l}\text { - partielle: } \\
\text { - grade IIa: } 3 \\
\text { - grade Ilb: } 1\end{array}$ & 4 & \\
\hline & & $\begin{array}{l}\text { - Aucune } \\
\text { - grade V: }\end{array}$ & 1 & \\
\hline & $\begin{array}{c}\text { Radiologique } \\
\text { (Consolidation) } \\
N=26\end{array}$ & $\begin{array}{c}\text { Satisfaisante } \\
(3-5 \text { mois })\end{array}$ & & 26 \\
\hline
\end{tabular}

Corp = corporectomie, $\mathrm{G}=$ greffe, $\mathrm{P}=$ plaque, $\mathrm{A}=$ agrafe (de $\mathrm{Blount}$ ), Erad. $=$ éradication simple.
Les conditions techniques locales ne permettaient pas de pratiquer systématiquement une ponction-biopsie.

La technique opératoire est indiquée dans le tableau 5. Le niveau lésionel était contrôlé sous amplificateur de brillance. Au niveau du rachis cervical inférieur la voie antéro-latérale droite, pré-sterno-cléido-mastoïdienne, fut utilisée, sous traction crânienne. Une corporectomie avec greffe iliaque autogène (3 cas) nous parut raisonnable dans les destructions corporéales importantes, à fortiori dans les écrasements (Figure la, b). L'adjonction d'une plaque vissée dans ces 3 cas prévenait l'éjection éventuelle du greffon. Une éradication des lésions complétée par une greffe intrafocale (technique de Hodgson) ${ }^{14}$ fut pratiquée dans deux cas d'ostéolyse C4-C5 (Figure 2a, b) et C5-C6, avec cyphose. Deux fois, une lésion débutante posant des problèmes de diagnostic différentiel justifia une exploration chirurgicale avec évacuation du pus et de séquestres disco-vertébraux ainsi qu'une biopsie. Après l'opération, le port d'une minerve plastique moulée fut imposé pendant 3 mois.

La laminectomie limitée ( 11 cas) et la laminotomie (3 cas) furent pratiquées dans les compressions postérieures dorsolombaires ( 10 cas) ou lombaires ( 4 cas) par abcès pottiques essentiellement, arachnoïdite ( 1 cas), ou une tuberculose vertébrale postérieure asssociée (l cas). Ces abcès étaient souvent de siège antéro-latéral.

Dans 5 cas, la lombotomie rétro-péritonéale gauche fut effectuée pour des lésions destructives (4 cas), mais aussi débutantes ( 1 cas) (Figure 3a, b, c).

Nous avons presque toujours réalisé des montages courts, ne pontant que la zone lésionnelle. Aucune immobilisation externe n'a été pratiquée au niveau dorso-lombaire.

\section{Resultats}

Le suivi post-opératoire clinique et radiologique des 26 patients varia de 6 à 108 mois: 6 mois ( 2 cas), 10 mois ( 3 cas), 12 mois ( 3 cas), 18 mois ( 6 cas), 24 mois ( 9 cas), 36 mois ( 2 cas), 108 mois ( 1 cas), soit une durée moyenne de 23 mois.

Aucune complication neurologique, mécanique ou septique à germe banal ne fut décelée. Tous les patients ont été déclarés guéris.

Les résultats à court et moyen termes sont indiqués dans le tableau 5. Sur les 4 paraplégies/tétraplégie de grade $\mathrm{V}$ et d'aggravation brutale, on observa une récupération complète, 2 récupérations partielles dont une reprise de la marche à l'aide d'une canne (grade IIa), mais avec spasticité, et une inchangée. Toutes les sciatalgies et brachialgies disparurent. Toutes les lésions ont consolidé entre 3 et 5 mois. La durée d'hospitalisation dans le service fut de 31 jours en moyenne (21 jours de chimiothérapie initiale et 10 jours après l'intervention). Ensuite, les patients présentant des troubles neurologiques furent transférés dans le service de rééducation fonctionnelle pour environ six semaines. Cette réhabilitation se poursuivit en externe.

\section{Discussion}

L'option thérapeutique dans le Mal de Pott chez l'adulte dépend, bien entendu, de l'expérience du chirurgien et des structures humaines et matérielles dont il dispose. Notre attitude chirurgicale est le fruit d'une réflexion approfondie et longuement mûrie. ${ }^{2}$ L'analyse objective dans l'ensemble des considérations matérielles, économiques et humaines difficiles, nous a 


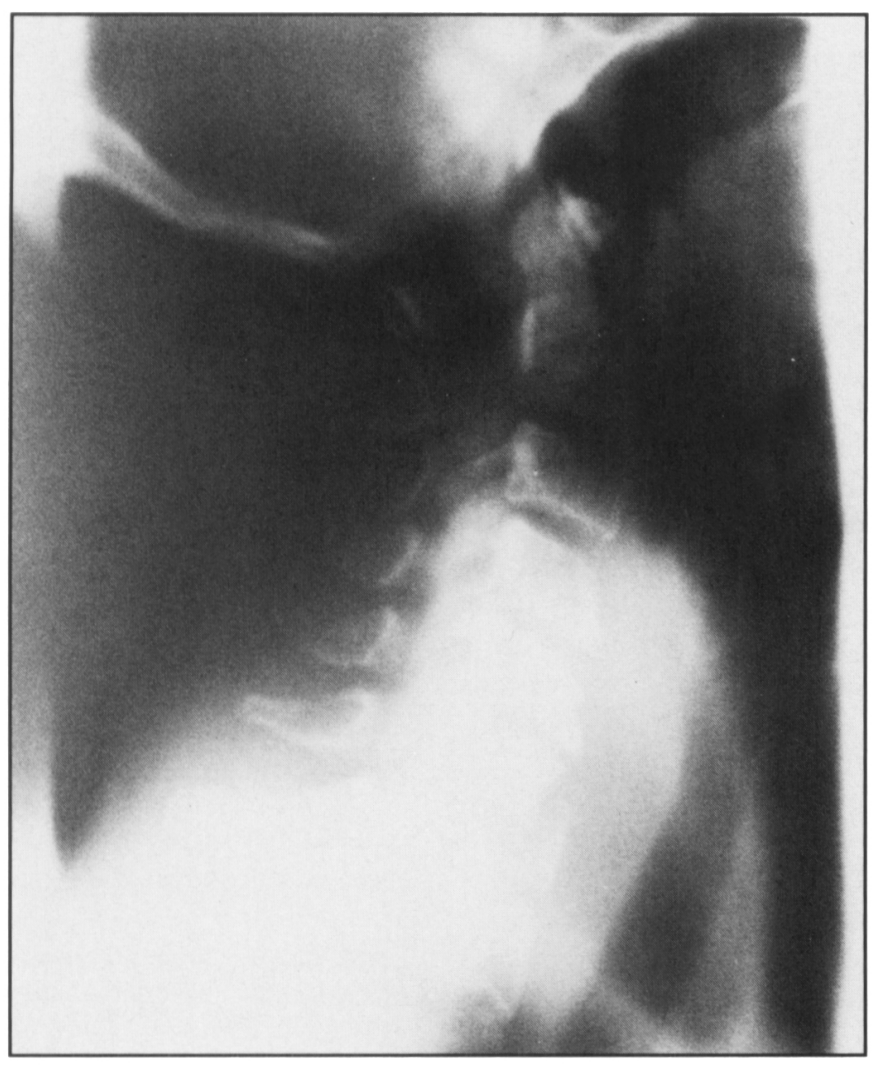

Figure Ja: Tomographie du rachis cervical, vue de profil: Mal de Pott C6-C7 avec écrasement de C7, érosion du plateau inférieur de C6.

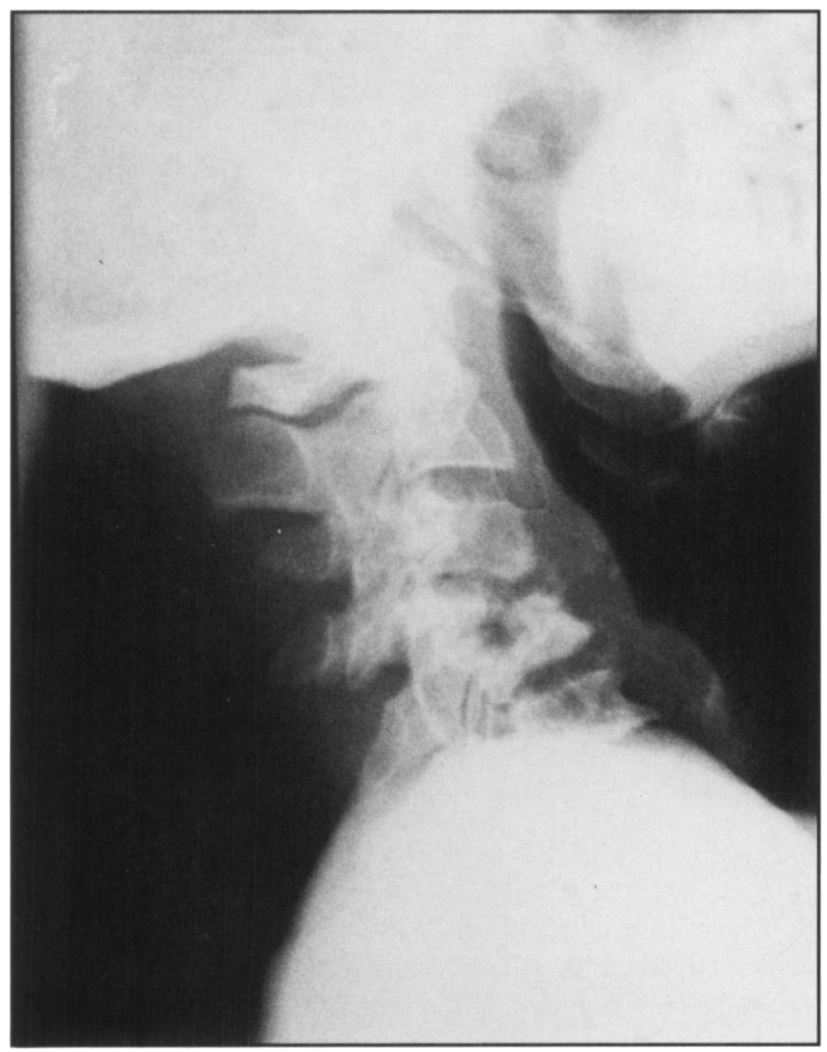

Figure 2a: Mal de Pott cervical avec ostéolyse de C4 et C5, et cyphose.

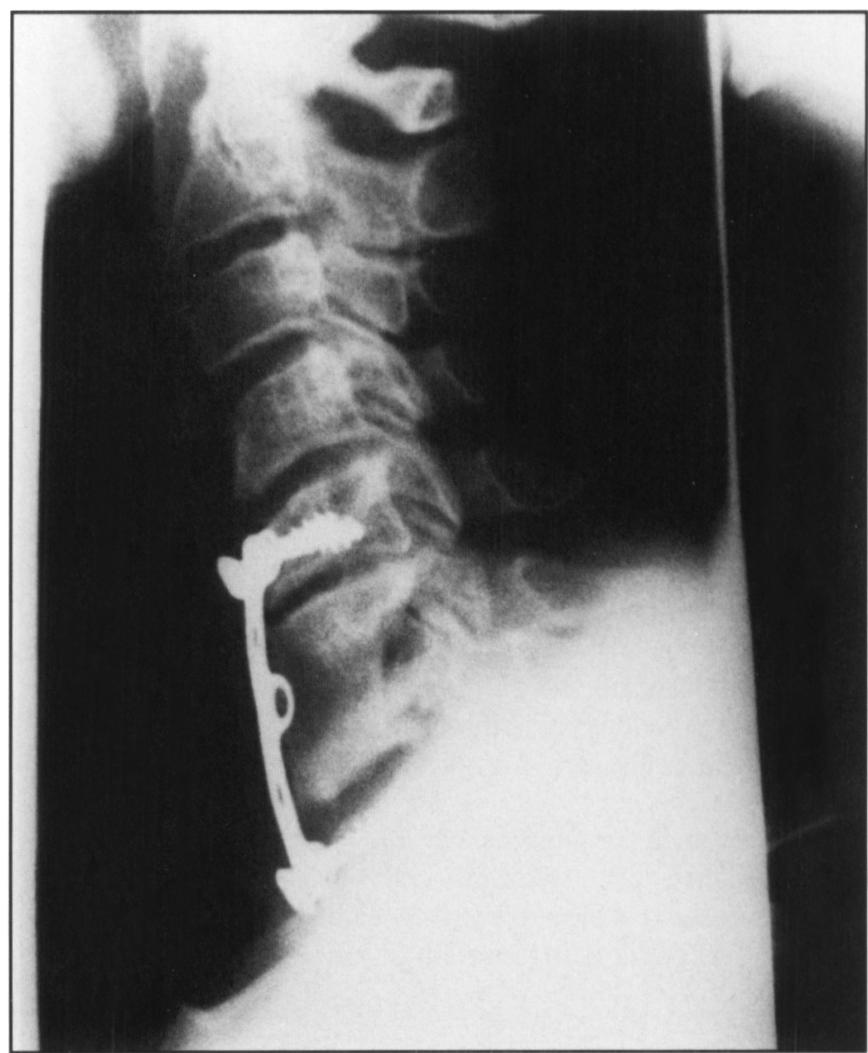

Figure 1b: Contrôle 8 mois après corporectomie de C7, corporectomie partielle de C6, greffe iliaque et plaque vissée: bonne fusion.

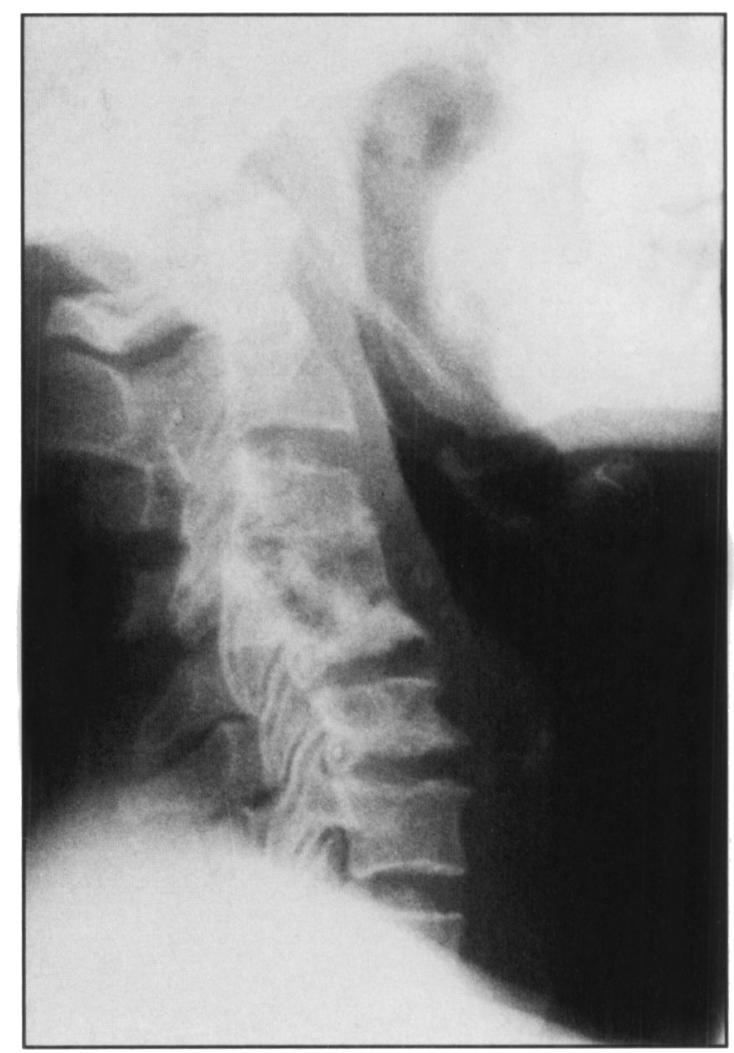

Figure 2b: Cliché 4 mois après la technique de Hodgson (C4-C5). Stabilité. 


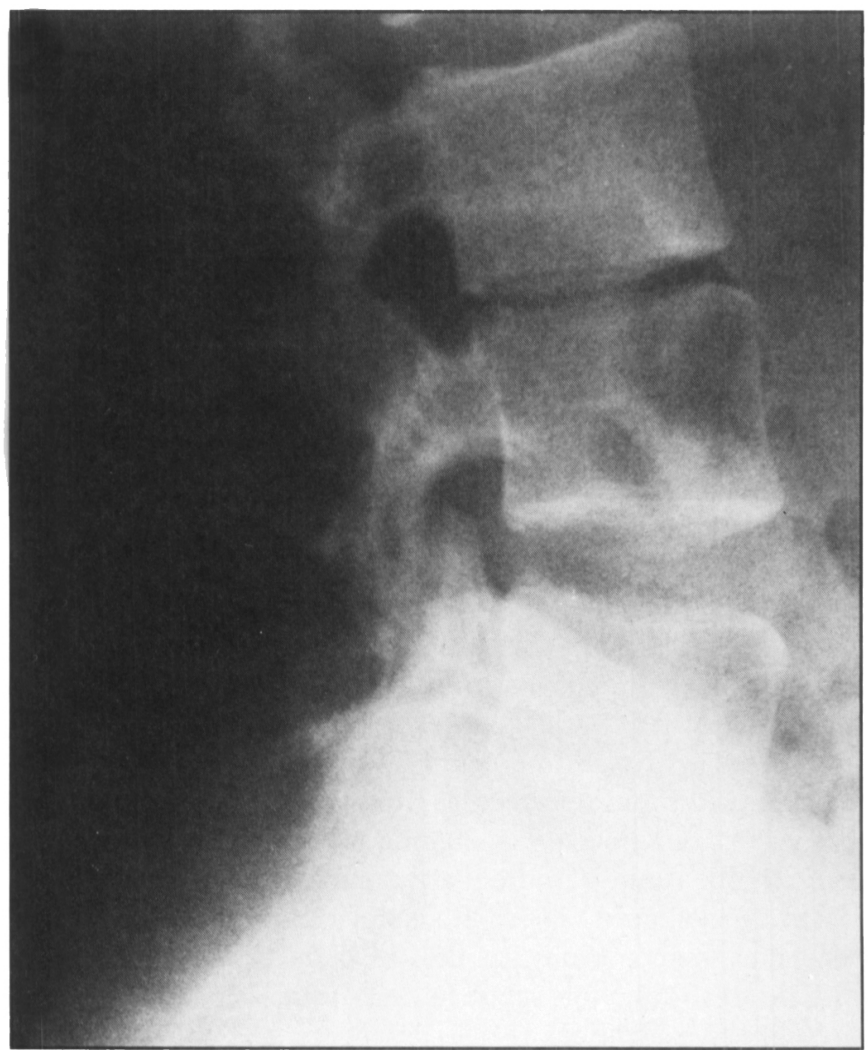

Figure 3a: Mal de Pott L3-L4 débutant: pincement L3-L4, discrète irrégularité des plateaux vertébraux.

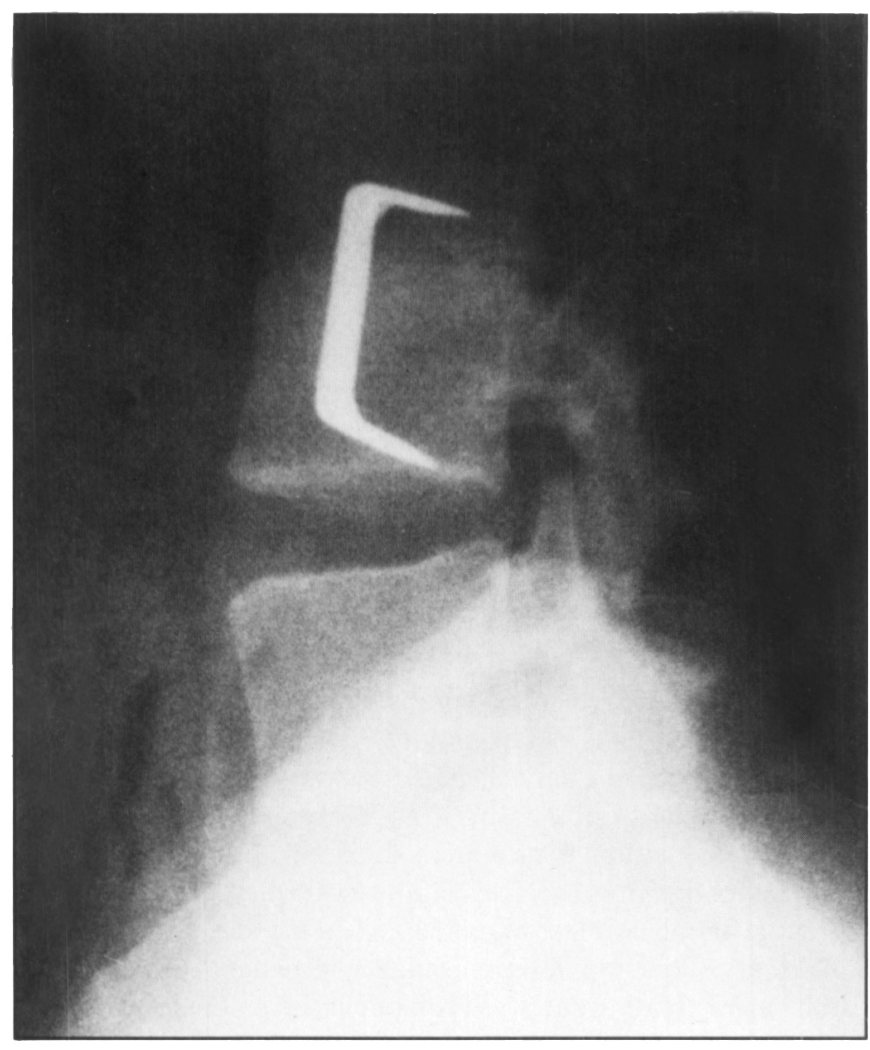

Figure 3c: Contrôle après un an. Fusion complète.

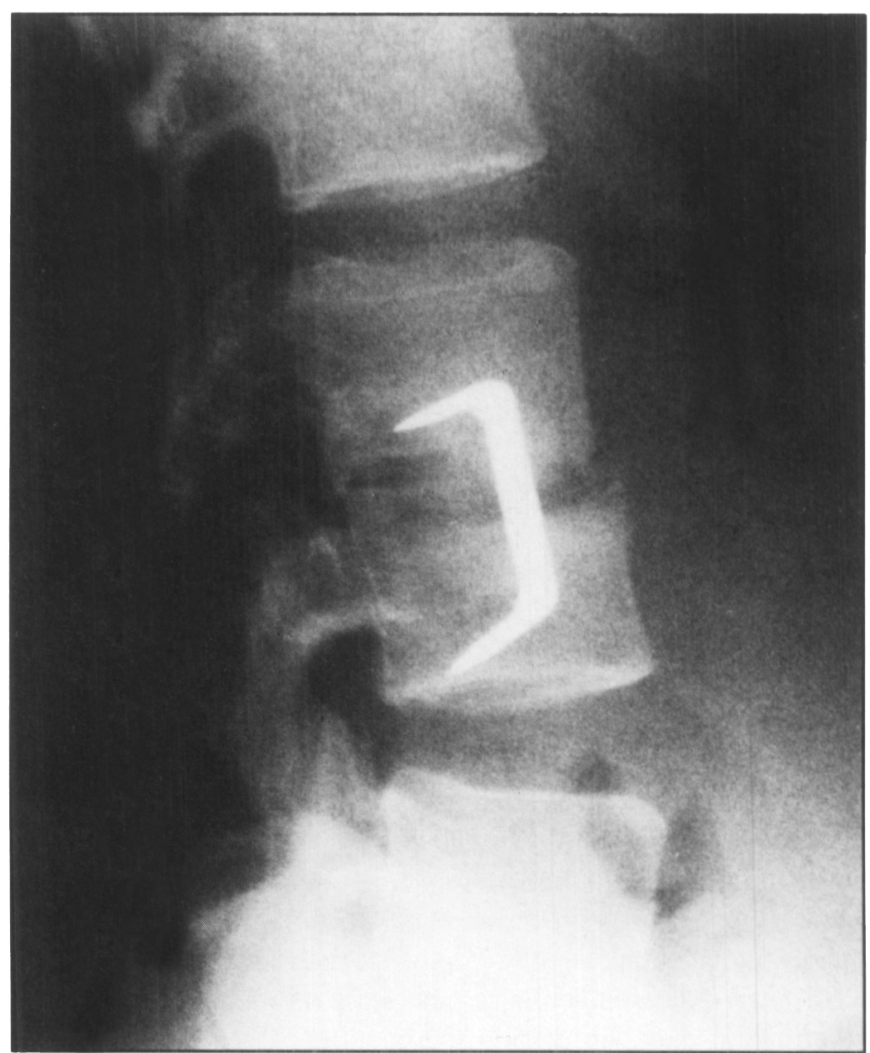

Figure 3b: Contrôle 2 mois après lombotomie rétropéritonéale - (technique de Hodgson et agrafe de Blount).

amenés à écourter au maximum la durée du traitement de la spondylodiscite tuberculeuse, en intervenant volontiers. L'étude de la littérature nous a confortés dans l'évolution de notre attitude thérapeutique, même si le traitement chirurgical reste à discuter en cas de tuberculose ostéo-articulaire.

Debeyre, ${ }^{5}$ en 1975 , notait que "jamais les chirurgiens n'abandonnèrent l'espoir de guérir le Mal de Pott par un acte chirurgical. Après les désastres provoqués, au début du siècle, par l'évacuation chirurgicale des abcès, la thérapeutique du Mal de Pott devint conservatrice, plus par raison que par conviction". Pour Aubry, ${ }^{9}$ en Afrique, I'intervention chirurgicale est habituelle dans le Mal de Pott, vue l'importance des lésions. C'est pour lui une indication de nécessité. Ouahes et Martini ${ }^{5.6}$ tempérèrent un peu en insistant sur la nécessité absolue de lier les indications opératoires aux structures humaines et matérielles de chaque hôpital. En d'autres lieux, Hodgson et al. ${ }^{14}$ ainsi que Griffiths ${ }^{13}$ abondent dans le même sens en précisant que la spondylodiscite tuberculeuse sans complication neurologique, doit être traitée par chimiothérapie associée à la chirurgie sous forme de l'opération de Hong Kong (ou technique de Hodgson) consistant en l'éradication des lésions complétée par une greffe antérieure intra-focale, ${ }^{14}$ à condition que l'on dispose d'une anesthésie et d'une réanimation adaptées et d'un personnel spécialisé et motivé.

Du point de vue orthopédique, l'association de la chimiothérapie et d'un geste chirurgical comprenant une éradication des lésions suivie d'une greffe focale, présente des avantages incontestables. 6.17 
Dans notre expérience, le traitement médical exclusif s'adresse aux lésions peu destructives, sans déformation ou instabilité rachidienne importante. Le problème du traitement d'un mal de Pott accompagné de signes neurologiques reste entier. ${ }^{6-8,16,17,20}$ La chimiothérapie est, bien sûr, indiscutable. Le geste chirurgical est par contre très discutable: il est sûrement utile dans certains cas, ${ }^{8.9 .12 .16}$ peut être nuisible dans d'autres. ${ }^{13.6 .7}$ Les tomographies informatisées et surtout la résonance magnétique nucléaire ${ }^{21}$ permettent au chirurgien de mieux déceler la cause de la paraplégie. Ces examens apportent ainsi des indications irremplaçables sur le type de stratégie chirurgicale adapté à chaque cas, en conditionnant en particulier le choix des voies d'abord, et la chronologie des différents temps opératoires en cas de double abord. Malheureusement, ces méthodes de diagnostic sont rarement à la disposition des chirurgiens qui travaillent dans les pays où la paraplégie pottique est encore fréquente et, à l'opposé, celle-ci est devenue rare dans les pays qui en disposent. Pattisson ${ }^{7}$ rapporta les résultats du traitement de 89 cas de paraplégies pottiques dont 85 traités par la chimiothérapie seulement: $84 \%$ de guérisons cliniques, $2 \%$ de décès et $13 \%$ d'échecs. Cet auteur insiste sur le fait qu'une aggravation des signes neurologiques au cours des premières semaines de chimiothérapie ( $48 \%$ des cas), ne doit pas être considérée comme une indication opératoire, car cette aggravation ne modifie pas fondamentalement le pronostic. Pour nous, la cause de la souffrance radiculomédullaire (abcès, os ou mixte) joue un rôle déterminant dans la décision thérapeutique. Les compressions d'origine osseuse ou mixte nous paraissent justifiables d'une approche médico-chirurgicale, donnant au patient le maximum de chance d'une récupération neurologique éventuelle, mais aussi pour une guérison avec stabilisation rachidienne rapide. Par ailleurs, l'aggravation brutale de la paraplégie est considérée par de nombreux auteurs comme une indication de décompression chirurgicale en urgence, ${ }^{8,12}$ les résultats étant habituellement satisfaisants, comme nous avons pu l'observer. Pour Martin, ${ }^{20}$ les paraplégies pottiques en Afrique et en Asie sont plus souvent dûes à des abcès compressifs. Cela explique probablement les bons résultats notés au décours du traitement médical exclusif.

Dans les formes évoluées, différentes affections peuvent simuler la spondylodiscite tuberculeuse, ${ }^{2.9 .12 .16}$ ce qui nécessite une approche diagnostique rationnelle, pour limiter ou éviter les erreurs de diagnostic par excès. La ponction-aspiration, guidée par tomographies informatisées, permet souvent de poser le diagnostic avec certitude et parfois de décomprimer la moelle en cas d'abcès. ${ }^{8}$ Par contre, dans notre centre, cette technique réalisée sous amplificateur de brillance, comporte plus d'inconvénients que d'avantages. Lorsqu'elle est non concluante ou non réalisable en toute sécurité, la biopsie chirurgicale peut être une alternative avantageuse. L'examen histopathologique, confronté aux contextes clinique et radiologique, permet de poser le diagnostic de tuberculose ostéo-articulaire dans près de $99 \%$ de cas. ${ }^{6}$ Par contre, la preuve bactériologique n'est pas toujours aisée. ${ }^{26.10}$ Ainsi, en milieu sous-équipé médicalement, le diagnostic de présomption reste parfaitement justifié; ${ }^{2.3}$ dans ces conditions, la chimiothérapie anti-tuberculeuse constitue un test à posteriori prouvant l'origine tuberculeuse de l'affection.

Nous n'avons pas d'expérience au niveau du rachis cervical supérieur $(\mathrm{C} 1, \mathrm{C} 2)$, localisation très rare $(0,3$ à $1 \%$ dans la littérature, 0 sur 160 cas dans notre propre série) $)^{6,10.11,18,19}$
(Loembé, Acta Neurochir (Wien) 1994, In press). Toutefois, une luxation $\mathrm{C} 1, \mathrm{C} 2$ isolée justifierait pour nous une arthrodèse postérieure plutôt qu'un traitement orthopédique, pour des raisons climatiques et la durée relativement longue de la phase de reconstruction. ${ }^{2}$ Fang et al. " préconisent par contre la voie transorale qui parait rationnelle et séduisante. L'éradication des lésions se fait par cette voie, l'arthrodèse pouvant être réalisée dans le même temps par insertion de greffon cortico-spongieux dans les tunnels creusés dans les massifs des apophyses articulaires. Cette arthrodèse peut aussi être pratiquée dans un second temps par voie postérieure. Bien qu'il s'agisse d'une intervention majeure nécessitant une équipe chirurgicale et de réanimation entraînées, elle semble fiable et performante, car les auteurs lui sont restés longtemps fidèles. Par ailleurs, en milieu neurochirurgical, les complications post-opératoires sont actuellement considérées négligeables, grâce à l'utilisation d'une instrumentation parfaitement adaptée (écarteur de Crockard), l'usage du microscope opératoire et de l'amplificateur de brillance, et le respect scrupuleux des règles d'asepsie. ${ }^{22}$

Au niveau cervical inférieur $(\mathrm{C} 3, \mathrm{C} 7)$, la voie pré-sterno-cléido-mastoïdienne droite classique nous parait simple et raisonnable. L'adjonction à la greffe d'une plaque vissée assure une stabilisation rachidienne immédiate. Sur nos 8 cas de fixation métallique (Tableau 5), dont 3 à la région cervicale, et 5 lombaires, nous n'avons pas déploré d'extension de l'infection initiale ni d'infection secondaire à germe banal. C'est pour prévenir cependant ces risques infectieux que nous réservons cette fixation métallique dans les formes peu ou non purulentes du Mal de Pott de l'adulte. D'autres auteurs ${ }^{10}$ préconisent, dans un premier temps, une ostéosynthèse postérolatérale par plaques de Roy-Camille, suivie d'un abord antérieur (technique de Hodgson).

Au niveau du rachis dorsal, nous intervenons le moins possible, les gibbosités gênantes devenant rares dans notre pays. Dans ces conditions, les lésions osseuses observées à ce segment rachidien n'entrainent pas d'instabilité appréciable, la cage thoracique tenant le tout en un bloc. En accord avec Ouahes et Martini, ${ }^{3}$ il est inutile d'opérer chez l'adulte les Maux de Pott s'accompagnant d'une cyphose de moins de 40 degrés, car l'accroissement possible de la cyphose reste très compatible avec un résultat fonctionnel et esthétique acceptable. C'est pourquoi nous donnons la préférence au traitement medical exclusif qui, en cas de trouble neurologique rebelle par abcès compressif, peut être associé à une laminotomie. Nous ne pratiquons actuellement la laminectomie que dans les rares cas de tuberculose des arcs postérieurs, ou d'arachnoïdite tuberculeuse associées.

Dans les cyphoses dorsales inférieures, de plus de 40 degrés, l'abord postérolatéral (costo-transversectomie) préconisé par Acikgoz et al. ${ }^{8}$ nous parait indiqué. Par contre au niveau thoracique supérieur et moyen, nous optons actuellement pour le traitement médical exclusif, la thoracotomie paraissant invasive,,$^{8,14,20}$ surtout dans notre contexte. ${ }^{3}$

L'instabilité lombaire d'origine pottique peut causer des douleurs incohersibles, associées ou non à des troubles neurologiques. Pour rapidement soulager le patient, une intervention chirurgicale peut s'avérer nécessaire. La lombotomie rétropéritonéale nous a donné satisfaction, les suites opératoires ayant été simples sur cette courte série, mais elle reste une intervention majeure. ${ }^{14}$ 
Depuis près de neuf ans, l'immobilisation au lit dans une coquille platrée ou l'immobilisation ambulatoire dans un corset platré n'a plus jamais été utilisée dans notre service, conformément aux données récentes de la littérature. ${ }^{3,6,23}$

Nous sommes conscients que dans un avenir proche, les efforts consentis par notre pays dans le domaine de la santé publique permettront certainement de réduire la fréquence de cette affection. ${ }^{2}$ Par ailleurs, il importe de la déceler précocement, pour éviter les complications osseuses et neurologiques invalidantes et favoriser ainsi le traitement médical exclusif.

Toutefois, dans les formes relativement évoluées du Mal de Pott, nous pensons que l'association d'un traitement médical court (quadrithérapie) et d'une chirurgie précoce, met le malade dans les meilleurs conditions pour une récupération neurologique éventuelle, mais aussi pour une guérison avec stabilisation rachidienne plus rapide. Cela permet aussi de préciser le diagnostic qui est souvent posé par excès, plus que par défaut.

\section{REFERENCES}

1. Auregan G, Bizeau F, Chakib S, Etienne B et Richard J. Le Mal de Pott à Djibouti. Analyse de 178 cas. Médecine et Armées 1990; 18: 3-6.

2. Loembe PM, Assengone-Zeh Y, Guerch M et Mbumbe-King A. La tuberculose vertébrale au Gabon. Neurochirurgie 1988; 34: 420427.

3. Ouahes M et Martini M. Résultat du traitement conservateur de 171 maux de Pott sans atteinte neurologique. Rev Chir Orthop 1988; 74: 563-568.

4. Panda M, Mukuna B et Ntungila N. Le mal de Pott et son traitement en milieu chirurgical zaïrois. Rachis 1992; 4: 177-182.

5. Debeyre J, et al. Mal de Pott. Traitement médical ou médico-chirurgical. (Table ronde). S.O.F.C.O.T., 49e Réunion annuelle, Rev Chir Orthop 1975; 61: 331 .

6. Martini M. La tuberculose ostéo-articulaire. Berlin: SpringerVerlag 1988; $200 \mathrm{p}$.

7. Pattisson PRM. Pott's paraplegia: an account of the treatment of 89 consecutive patients. Paraplegia 1986; 24: 77-91.
8. Acikgoz B, Ozcan OE, Belen D, Erbengi A et Ozgen T. Surgery for progressive Pott's paraplegia (Tuberculous paraplegia). Paraplegia 1991; 29: 537-541.

9. Aubry P, Capdevielle P et Durand G. Les tuberculoses extra-pulmonaires de l'Africain. Méd Trop 1979; 39: 157-163.

10. Borne G, Bedou G, Cristino G et Hussein A. Mal de Pott cervical. Considérations techniques à propos d'un cas opéré. Neurochirurgie 1988; 34: 120-122.

11. Fang D, Leong JCY, Fang HSY. Tuberculosis of the upper cervical spine. J Bone Joint Surg (Br) 1983; 65: 47-50.

12. Ferro RM, Barnaud $P$ et Carayon $A$. Le Mal de Polt chez l'Africain. Méd Trop 1979; 39: 191-208.

13. Griffiths DLI. The treatment of spinal tuberculosis. Recent Adv Orthop 1979; 3: 1-17.

14. Hodgson AR, Stock FE, Fang HST et Ong CB. Anterior spinal fusion. The operative approach and pathological findings in 412 patients with Pott's disease of the spine. Brit J Surg 1960;48: 172-178.

15. Lifeso RM, Weaver $\mathrm{Ph}$ et Harder EH. Tuberculous spondylitis in adults. J Bone Joint Surg (Am) 1985; 67: 1405-1413.

16. Louis R, Conty CR et Pouye I. Tuberculose osseuse et articulaire de l’Africain de l'Ouest. Méd Afr Noire 1969; numéro spécial: 65-87.

17. Medical Research Council. A 10 year assessment of a controlled trial comparing debridment and anterior spinal fusion in the management of tuberculosis of the spine in patients on standard chemotherapy in Hong Kong. J Bone Joint Surg (Br) 1982; 64: 392-398.

18. Stecken J, Boissonet H, Manzo L, Pheline C, Dobbelaere P et Yaffi D. Mal de Pott sous occipital. Neurochirurgie 1987: 33: 483-486.

19. Wang LX - Peroral focal debridment for treatment of tuberculosis of the atlas and axis. Chin J Orthop 198I; I: 207-209.

20. Martin NS. Pott's paraplegia: a report of 120 cases. J Bone Joint Surg (Br) 1971; 53: 596-608.

21. Roche PH, Malca SA et Pellet W. Spondylodiscite tuberculeuse. Eléments du diagnostic et intérêt de l'IRM. A propos d'une localisation cervicale. Neurochirurgie 1993; 39: 248-253.

22. Galibert P, Toussaint P, Le Gars D, Rosat P et Quequet M. La voic trans-orale. Rachis 1981: 1: 422-430.

23. Medical Research Council. A 10 years assessment of controlled trials of inpatient and outpatient treatment and of plaster-of-paris jackets for tuberculosis of the spine in children on standard chemotherapy. J Bone Joint Surg (Br) 1985; 67: 103-110. 